\title{
Extinction Limits of Premixed Combustion Assisted by Catalytic Reaction in a Stagnation-Point Flow
}

\author{
Jingjing Li and Hong G. Im* \\ Mechanical Engineering, University of Michigan, Ann Arbor, Michigan, 48105
}

\begin{abstract}
Motivated by the potential use of catalytic materials in micro-combustor application, the primary scope of this study is to investigate the lean extinction characteristics of premixed flames in the presence of catalytic reaction. In particular, the effects of mixture dilution on the lean flammability limit are examined by adopting a stagnation-point flow system with a methane/air mixture over a platinum surface. For the reference fuel/air conditions, it was found that the lean flammability limit of homogeneous reaction is actually lower than that of the heterogeneous reaction. However, when sufficient amount of nitrogen is added to the reactant mixture, the surface reaction shows the benefit of extending the lean extinction limit to a lower value. It is also found that the magnitude of flammability extension by surface reaction is more pronounced in the presence of surface heat loss. Unsteady extinction behavior in response to equivalence ratio fluctuations shows an overall trend consistent with the steady results. These phenomena are explained as result of different characteristic time scale of homogeneous and heterogeneous reaction. Analytical calculation proved that under same reactant and thermal boundary condition, surface reaction has smaller time scale which is rather insensitive to dilution while gas phase reaction has larger one which changes more with different dilution magnitude.
\end{abstract}

\section{Introduction}

Development of micro-power-generation systems with a dimension ranging from a few centimeters to a fraction of a millimeter has recently attracted strong research interests in favor of their high energy density and portability. For this type of combustors, high combustion temperature is undesirable since it adversely affect the mechanical and chemical structures of the interior parts. This problem can be alleviated by achieving low-temperature combustion using lean reactant mixtures. Other benefits of lean combustion include higher combustion efficiency and lower pollutant emission such as NOx or soot. However, low temperature combustion is more difficult to achieve in micro-combustors because their high surface-to-volume ratio causes a large heat loss compare to macro-scale combustors. A feasible approach to overcome this difficulty is to employ surface reaction using a catalytic material to assist stable combustion. It is therefore of fundamental importance to assess the! extent of flammability enhancement due to the surface reaction, especially under lean mixture conditions.

${ }^{*}$ Corresponding author. Tel.: 734-615-5152; fax: 734-647-3170; hgim@ @umich. 
Flammability extension by catalytic reaction has been studied in various configurations. Park et al. ${ }^{1}$ performed a numerical study of hydrogen/air stagnation-point flow model with a platinum catalyst, and showed that catalytic reaction can extend both lean and rich flammability limits. Seo et $a .^{2}$ experimentally investigated a channel flow combustor with a paladium catalyst to determine the minimum surface temperature for sustained combustion. Their results at a flow velocity of $23.2 \mathrm{~m} / \mathrm{s}$ showed that, when the fuel-air ratio was $4.25 \%$, the preheat temperature at which the flame extincts was lower than the preheat temperature of surface extinction. When the fuel-air ratio was $3.86 \%$ or less, the preheat temperature for flame extinction was higher than that of surface extinction. These results suggest that successful application of catalyst-assisted microcombustion depends on parametric conditions of the combustion systems.

Another issue of fundamental interest is the effect of unsteadiness. Due to the small dimension, micro-combustors are more likely to be subjected to fluctuations in the system parameters such as the flow rate, composition, and surface heat loss. It has also been understood that flames near extinction conditions are in general more sensitive to external perturbations, ${ }^{3}$ which can sometimes lead to combustion instability. Therefore, the flammability extension by catalytic reactions must be carefully examined under unsteady conditions. A number of studies exist on the response of strained flames to unsteady strain rate ${ }^{3-8}$ and composition fluctuations. ${ }^{9,}{ }^{10}$ In this study, these issues are investigated in a system with homogeneous/heterogeneous reactions. In particular, the concept of the dynamic flammability limit ${ }^{10}$ and its extension due to catalytic reaction! s are explored.

Therefore, the objective of the present study is to conduct numerical simulations of a stagnationpoint flow system with a methane-air mixture over a platinum surface. The effects of mixture dilution and surface thermal conditions on the lean extinction limit are examined for a steady system. Subsequently, combustion behavior in response to unsteady equivalence ratio is investigated in the context of the flammability extension by catalytic reaction.

\section{Model Description}

As shown in Fig. 1, the computational configuration is a stagnation-point flow where a mixture of methane and oxidizer is injected onto a platinum surface, forming a flame either in the flow field or on the catalytic surface. One-dimensional similarity formulation is derived with full consideration of detailed surface ${ }^{11}$ and gas-phase ${ }^{12}$ chemical kinetic models. As in our previous study, ${ }^{13}$ both steady and unsteady computations are performed by a modified version of OPUS ${ }^{14}$ to incorporate solid wall and surface chemistry. A fixed zonal grid refinement is applied to provide sufficient resolution in the reaction zone near the solid surface. For all calculations, the inlet gas temperature and pressure is fixed at $300 \mathrm{~K}$ and $1 \mathrm{~atm}$, respectively. The distance between the inlet and the catalytic surface is $L=0.5 \mathrm{~cm}$.

One of the main issues in this study is the effects of dilution on the separation of characteristic time scales for gas-phase and surface reactions. For this purpose, additional nitrogen is mixed into the air, measured by its mole fraction. For example, $10 \%$ nitrogen adddition indicates that 0.1 mole nitrogen is added to 1 mole of air.

To study unsteady flame response, the reactant composition is varied in time according to the following equations:

$$
X_{\mathrm{CH}_{4}}(t)=X_{\mathrm{CH}_{4}}(0)[1-A(1-\cos (2 \pi f t))] \text {, }
$$

where $X$ is the mole fraction at inlet. The strain rate is then defined as the nominal velocity gradient, $S=U_{\infty} /(2 L)$, due to the difficulty in defining the actual velocity gradient when the 
reaction occurs on the surface. ${ }^{13}$

\section{Results and Discussion}

\section{A. Steady response: effects of dilution}

As a baseline study, the steady flame behavior subjected to reactant mixture dilution is first investigated, as an attempt to assess the effect of surface chemistry on extending the lean extinction limit at various level of gas mixture dilution. In general, it is anticipated that dilution lowers temperature and hence the reaction rate, such that the flame extinction occurs at a higher equivalence ratio. It is of interest to examine whether this effect applies to both homogeneous and heterogeneous reactions at a comparable level.

Figure 2 shows the maximum reaction zone temperature as a function of the mixture equivalence ratio for various nitrogen dilution levels, each case with and without surface reaction. For all the calculations, the strain rate was fixed at $277 \mathrm{~s}^{-1}$ and the surface was considered adiabatic. The results for all cases show that the maximum temperature decreases as the equivalence ratio is reduced, eventually reaching the lean extinction limit. As expected, it is clearly seen that an increased amount of dilution lowers the reactivity such that extinction occurs at higher equivalence ratio. An important observation here is that the disparity between the lean extinction limit of homogeneous and heterogeneous combustion becomes larger as the level of dilution is increased. For example, in $0 \%$ dilution case the extinction limit for the homogeneous reaction is lower than that for the heterogeneous case. With $13 \%$ dilution, however, the heterogeneous comb! ustion shows a much wider flammable range of the equivalence ratio. This implies that the sensitivity in the reduction of the characteristic reaction time scales to the mixture dilution is different between homogeneous and heterogeneous combustion processes. Therefore, it is expected that the benefit of flammability limit extension by catalytic reaction is greater as the reactant mixture is more diluted. This behavior was found to be qualitative consistent for other values of the strain rate.

More extensive calculations were performed to determine the extinction limit extension over a range of nitrogen dilution. In addition to adiabatic wall considered in the above, we have also considered the cases where the catalytic surface is subjected to heat loss or heat addition. Heat loss from the surface is modeled by a general form: $\dot{q}=h\left(T_{\text {surf }}-T_{0}\right)$, where $T_{0}$ is the ambient temperature set at $300 \mathrm{~K}$, and $h$ is the effective heat transfer coefficient accounting for the associated conductive and conductive modes. In this study, $h=4 \mathrm{~W} / \mathrm{m}^{2}-\mathrm{K}$ is used. For the heated surface case, a net power of $10^{4} \mathrm{~W} / \mathrm{m}^{2}$ is supplied to the substrate.

Figure 3 (a) is plot of lean extinction limits as a function of the nitrogen dilution for various thermal conditions on the surface. To show the result more clearly, (b) is differences of extinction equivalence ratio between with and without surface reaction in (a). For all surface conditions, it is interesting to note that catalytic reaction results in higher lean extinction limit. In other words, catalytic reaction actually leads to a narrower flammable range. The benefit of flammability extension by surface chemistry occurs only with nitrogen dilution of approximately $5 \%$ and above, and the level of extension continues to increase as the level of dilution increases. This behavior is attributed to the fact that, at such lean conditions, the addition of catalytic surface scavenges radicals generated from the gas-phase reactions, hence attenuating the overall reactivity of the system. This results provide an important practical implication that the $u$ ! se of surface reaction in a lean premixed micro-combustor may be applicable only with a significant level of dilution, which is rather a desirable feature for the purpose of achieving low temperature combustion. 
Figure 3 also demonstrates that, while heat loss or heat supply on the surface can significantly affect the absolute level of the lean extinction limits, the difference between the homogeneous and heterogeneous extinction limits are largely unaffected by the surface thermal conditions. It is also noted that the crossover point between the homogeneous and heterogeneous limits (x coordinate value when y equals zero in Figure 3 (b)), albeit marginally, tends to decrease slightly toward a lower level of dilution with heating surface. This is because surface reaction can be more effectively enhanced by heat supply than homogeneous reaction.

The effect of nitrogen dilution is primarily to lower the flame temperature and thereby lengthening the characteristic chemical time scales relative to the characteristic flow residence time. It is apparent that this effect is more pronounced in the gas-phase than on the surface. To verify this observation, the characteristic chemical time scales for the gas-phase and surface chemistry, $\tau_{g}$ and $\tau_{s}$ respectively, are defined based on the primary fuel consumption as ${ }^{13}$

$$
\begin{gathered}
\tau_{g}=\frac{X_{\mathrm{CH}_{4}}}{\dot{\omega}_{\mathrm{CH}_{4}}} \frac{p}{R^{0} T}, \\
\tau_{s}=\frac{X_{\mathrm{CH}_{4}} \Gamma}{\dot{\omega}_{\mathrm{CH}_{4}}},
\end{gathered}
$$

where the units are in seconds, $X_{\mathrm{CH}_{4}}$ is mole fraction of methane at the nozzle exit, $R^{0}$ is the universal gas constant, and $\Gamma$ is site density of the surface species. In determining the reaction rate, $\dot{\omega}_{\mathrm{CH}_{4}}$ for the surface reactions is evaluated at the catalytic surface, while for the gas-phase reactions the spatial maximum value of $\dot{\omega}_{\mathrm{CH}_{4}}$ is used. Note that $\dot{\omega}_{\mathrm{CH}_{4}}$ in Eqs. 2 and 3 have different units. ${ }^{13}$ The characteristic times are evaluated at a fixed strain rate of $S=277 \mathrm{~s}^{-1}$ and adiabatic surface.

The results for the three dilution cases considered in Fig. 2 are summarized in Table 1. It is found that the gas-phase reaction time scales increases with dilution by several factors, while the surface reaction time scale remains almost the same. This confirms that the gas-phase reaction is more sensitive to dilution than surface reaction.

\section{B. Dynamic flammability limit}

To further examine the effects of dilution on unsteady flame characteristics, we now study the unsteady flame response to oscillatory equivalence ratio as given by Eq. (1). The main interest is to identify the extension of lean flammable limit for a given strain rate as a function of frequency, following the concept of the dynamic flammability limit, which is defined as the minimum instantaneous equivalence ratio during the oscillation without extinction, introduced by Sankaran and Im. ${ }^{10}$ For each frequency, $f$, we can determine the maximum allowable amplitude, $A$, beyond which the flame extinguishes. This implies that the dynamic flammability limit is a function of frequency for the given strain rate, surface thermal condition, and the initial equivalence ratio. We attempt to conduct an analysis to a system in the presence of heterogeneous combustion, with an emphasis on the effects of nitrogen dilution, heat loss, and the frequency of the equivalence ratio oscillation.

The flame responses with and without catalytic reaction are compared under three cases: (a) $0 \%$ dilution without heat loss, (b) $13 \%$ dilution without heat loss, and (c) $13 \%$ dilution with heat loss. Following Eq. (1), the initial condition, $X_{\mathrm{CH}_{4}}(0)$, as been set such that the equivalence ratio, $\phi=2 X_{\mathrm{CH}_{4}}(0) / X_{\mathrm{O}_{2}}(0)$, is $0.68,0.76$, and 0.80 for cases (a) to (c), respectively. For all cases, the strain rate is set to $277 \mathrm{~s}^{-1}$. 
Figures 4(a) to (c) shows the limit-cycle behavior of the maximum flame response for various frequencies of oscillation in $\phi_{\text {flame }}$, which is determined at the upstream flame base at $T=302$ K. Since the amplitude of $\phi_{\text {flame }}$ was taken to be the maximum without quenching the flame, the minimum equivalence ratio reached during the limit cycle is considered the dynamic flammability limit for a given frequency condition. Comparing Figs. 4(a) and (b), it is clear that the diluted case shows a greater extension of the lean flammability limit by adding the surface reaction; not only is the steady flammability limit further extended to a leaner condition, but the additional unsteady extension for the same frequency is also greater with surface reaction. This is attributed to the fact that the surface reaction is faster and thus can respond to the imposed oscillation more rapidly. Moreover, the surface reaction case und! ergoes a larger excursion in the flame strength, hence there is a larger amount of excess enthalpy during a cycle to sustain combustion at leaner conditions. Similar behavior is also observed in Fig. 4(c) in the presence of heat loss, although the overall flammability limits are relatively higher than those in (b). As discussed in Fig. 3, the amount of flammability extension due to surface reaction is found to be rather insensitive to the surface thermal condition even considering unsteady situations.

To summarize the unsteady characteristics, it is of interest to compare the dynamic flammability extension between the cases with and without surface reaction, denoted by $\Delta \phi_{s}$ and $\Delta \phi_{g}$, respectively. Figure 5 shows the dynamic flammability limits extension for with/without surface reaction and their difference, $\Delta \phi_{s}-\Delta \phi_{g}$, as a function of frequency for the three cases shown in Fig. 4. Note that the increase in the curve with frequency in Figure 5 (b) indicates that the additional dynamic flammability extension due to surface reaction becomes greater as frequency increases. In other words, the benefit of surface reaction in extending flammability is greater for a larger level of unsteadiness. For $0 \%$ dilution, the curves fall into negative region in (b) due to the fact that surface reaction acts negatively on the system flammability. However, there is a reversal in ! the curve at high frequencies, resulting in flammability enhancement by surface reaction even with $0 \%$ dilution. Therefore, the benefit of catalytic flammability extension is found to be greater with dilution, while the difference between the cases with and without heat loss appears to much less significant.

\section{Conclusions}

In this paper, steady and unsteady extinction characteristics of a stagnation-point flow combustor were analyzed with both homogeneous (methane-air) and heterogeneous (platinum) reactions considered. One of the key aspects of the study was the effect of mixture dilution on the aspects of flammability extension. For the system under study, it was found that surface reactions actually attenuates the range of lean flammbility limit due to their effect of radical scavenging. The benefit of catalytic reactions can indeed occur and it increases with additional dilution in the gas mixture, which is a desirable aspect in achieving low temperature combustion for micro-combustor application. While the surface heat loss expectedly lowers the overall flammability of the system, it was found that the level of flammability extension by surface reactions is rather insensitive to the surface thermal conditions.

Unsteady extinction behavior in response to oscillatory strain rate and equivalence ratio fluctuations showed overall consistent trend expected from the steady results. The overall flame response becomes attenuated as the frequency of oscillation increases, and the cut-off frequency depends on the characteristic time scales of the dominant chemical reactions. It was also found that the 
benefit of flammability extension by catalytic reactions can be greater as the level of unsteadiness increases. These results can provide insights into the development of robust micro-combustion devices.

\section{Acknowledgments}

This work was supported by the National Science Foundation Grant CTS-0134128 under the technical monitoring of Dr. Linda Blevins. The authors would like to thank Dr. Ramanan Sankaran of Sandia National Laboratories for his contributions in the development of the numerical methods used in this study.

\section{References}

${ }^{1}$ Park, Y. K., Bui, P-A, Vlachos, D. G., ’Operation Regimes in Catalytic Combustion: H2/Air Mixtures Near Pt", AIChE J., Vol. 44, No. 9,(1998) pp. 2035-2043.

${ }^{2}$ Seo, Y. S., Kang, S. K., Shin, H. D., "Catalytic Combustion of Lean Premixed Mixture in Catalytically Stabilized Thermal Combustor", Combust. Sci. Tech., Vol. 145, No. 1-6, 1999, pp. 17-35.

${ }^{3} \mathrm{Im}$, H. G., Law, C. K., Kim, J. S., Williams, F. A., "Response of Counterflow Diffusion Flames to Oscillating Strain Rates", Combust. Flame, Vol. 100, No. 1-2, 1995, pp. 21-30.

${ }^{4}$ H.G. Im, J.K. Bechtold, C.K. Law, "Counterflow Diffusion Flames with Unsteady Strain Rates", Combust. Sci. Tech., Vol. 106, 1995, pp. 345-361.

${ }^{5}$ Im, H. G., Bechtold, J. K., Law, C. K., "Response of Counterflow Premixed Flames to Oscillating Strain Rates", Combust. Flame, Vol. 105, 1996, pp. 358-372.

${ }^{6}$ Egolfopoulos, F. N., Campbell, C. S., "Unsteady Counterflowing Strained Diffusion Flames: Diffusion-limited Frequency Response", J. Fluid Mech., Vol. 318, 1996, pp. 1-29.

${ }^{7}$ Ghoniem, A. F., Soteriou, M. C., Knio, O. J., "Effect of Steady and Periodic Strain on Unsteady Flamelet Combustion"Proc. Combust. Inst., Vol. 25, 1992, pp. 223-230.

${ }^{8}$ Sung, C. J., Law, C. K., "Structural Sensitivity, Response, and Extinction of Diffusion and Premixed Flames in Oscillating Counterflow"Combust. Flame, Vol. 123, No. 3, 2000, pp. 375-388.

${ }^{9}$ Lauvergne, R., Egolfopoulos, F. N., "Unsteady Response of C3H8/Air Laminar Premixed Flames Submitted to Mixture Composition Oscillations", Proc. Combust. Inst., Vol. 28, No. 2, 2000, pp. 1841-1849.

${ }^{10}$ Sankaran, R., Im, H. G., ’Dynamic Flammability Limits of Methane/Air Premixed Flames with Mixture Composition Fluctuations", Proc. Combust. Inst., Vol. 29, No. 1, 2002, pp. 77-84.

${ }^{11}$ Deutschmann, O., Schmidt, R., Behrendt, F., Warnatz, J., "Numerical Modeling of Catalytic Ignition", Proc. Combust. Inst., Vol. 26, 1996, pp. 1747-1754.

${ }^{12}$ Smith, G. P., Golden, D. M., Frenklach, M., Moriarty, N. W., Eiteneer, B., Goldenberg, M., Bowman, C. T., Hanson, R. K. Hanson, Song, S., Gardiner, W. C., Lissianski, V. V., Qin, Z., http://www.me.berkeley.edu/gri mech.

${ }^{13} \mathrm{Li}$, J., Im, H. G., "Extinction Characteristics of Catalyst-Assisted Combustion in a Stagnation-Point Flow Reactor", Combust. Flame, in press, 2005.

${ }^{14}$ Im, H. G., Raja, L. L., Kee, R. J., Lutz, A. E., Petzold, L. R., ”Opus: A Fortran Program for Unsteady OpposedFlow Flames", Report No. SAND2000-8211, Sandia National Laboratories, 2000.

${ }^{15} \mathrm{Im}$, H. G., Chen, J. H., "Effects of Flow Transients on The Burning Velocity of Laminar Hydrogen/Air Premixed Flames", Proc. Combust. Inst., Vol. 28, No. 2, 2000, pp. 1833-1840. 
Table 1. Time scales of gas-phase and surface fuel consumption rates for various levels of dilution.

\begin{tabular}{c|c|c|c}
\hline & $0 \% \mathrm{~N}_{2}$ & $9 \% \mathrm{~N}_{2}$ & $13 \% \mathrm{~N}_{2}$ \\
\hline$\tau_{g}$ & $1.365 \times 10^{-4} \mathrm{~S}$ & $2.076 \times 10^{-4} \mathrm{~S}$ & $2.490 \times 10^{-4} \mathrm{~s}$ \\
$\tau_{s}$ & $9.201 \times 10^{-6} \mathrm{~S}$ & $8.696 \times 10^{-6} \mathrm{~s}$ & $8.637 \times 10^{-6} \mathrm{~s}$ \\
$\tau_{g} / \tau_{s}$ & 14.8 & 23.9 & 28.8 \\
\hline
\end{tabular}

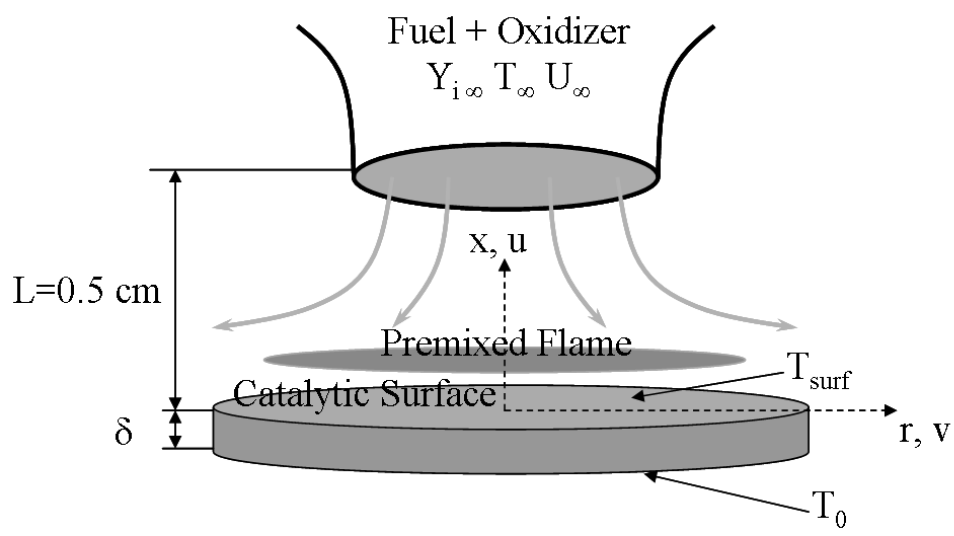

Figure 1. Schematic of the problem configuration.

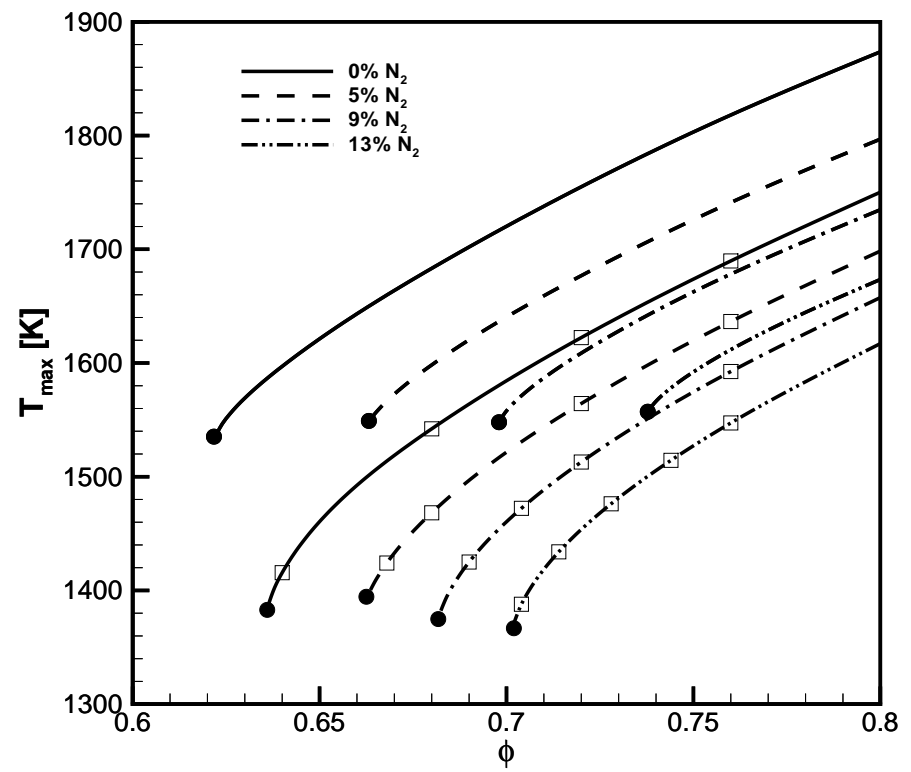

Figure 2. Maximum temperature as a function of the equivalence ratio for various levels of nitrogen dilution for adiabatic surface. Lines without symbols: without surface reactions. Lines with symbols: with surface reactions. 


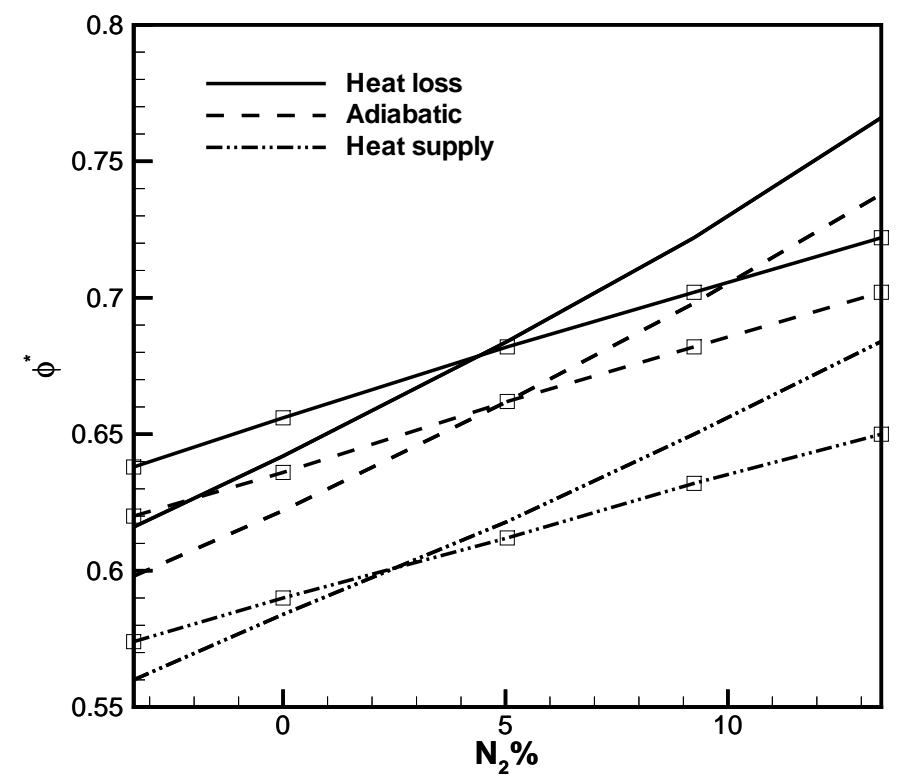

(a)

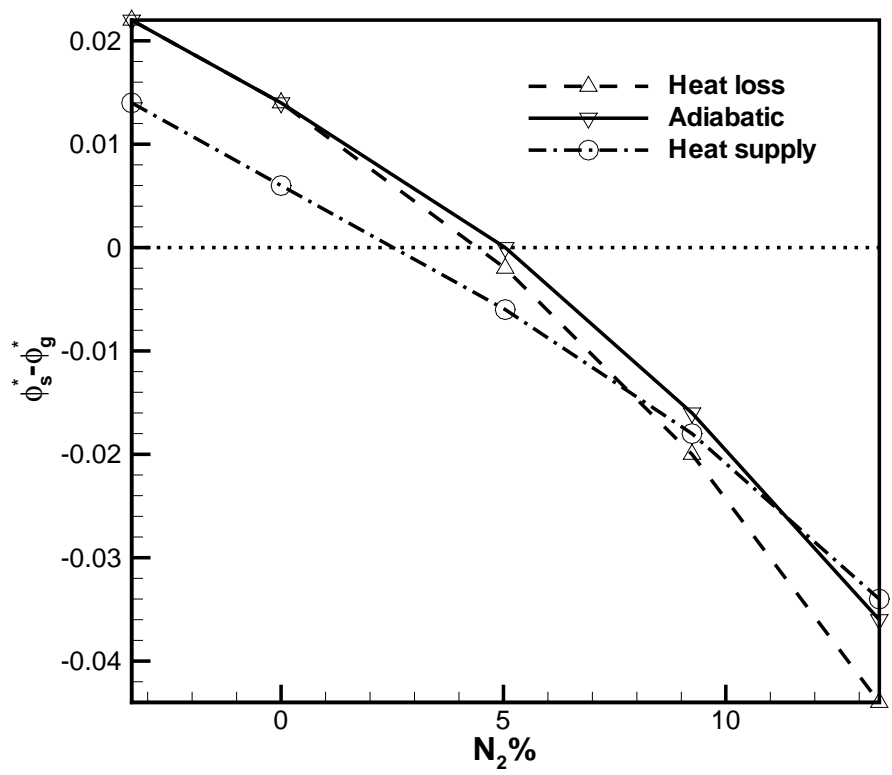

(b)

Figure 3. Lean extinction limits and their differences between with and without surface reaction as a function of the nitrogen dilution for various surface thermal conditions, for $S=277 s^{-1}$. For (a), lines without square symbols: without surface reactions. Lines with square symbols: with surface reactions. 


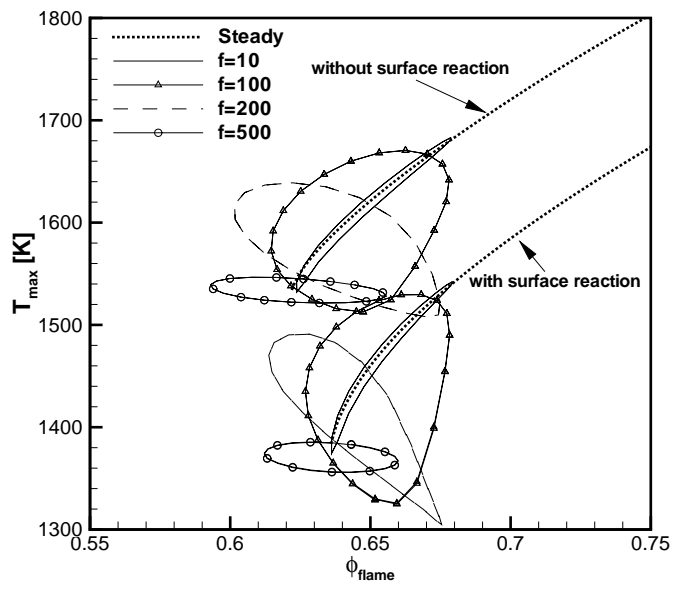

(a) $0 \%$ dilution without heat loss

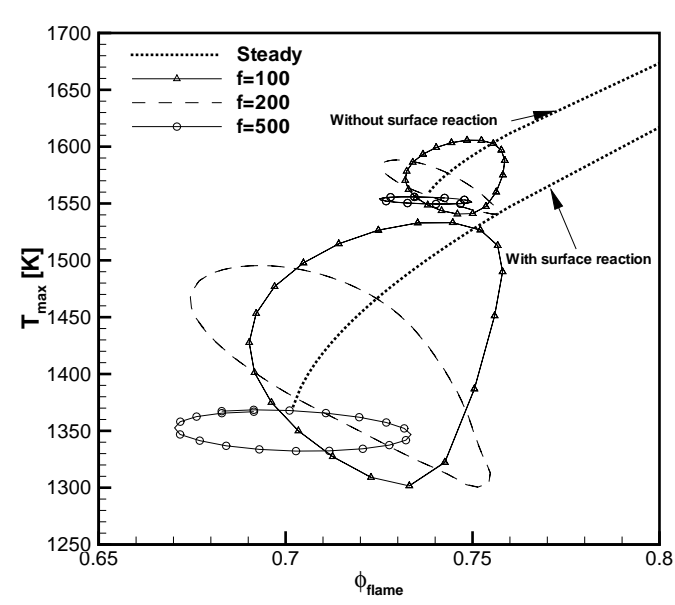

(b) $13 \%$ dilution without heat loss

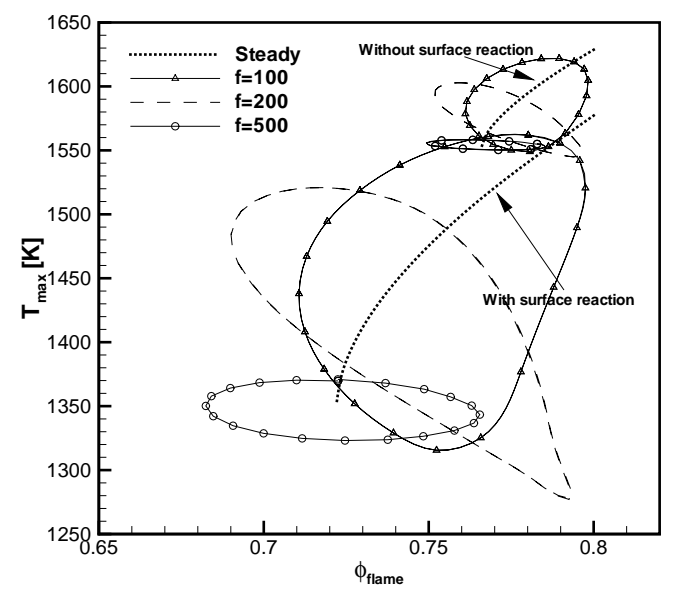

(c) $13 \%$ dilution with heat loss

Figure 4. Unsteady flame temperature response to the equivalence ratio various frequencies. 


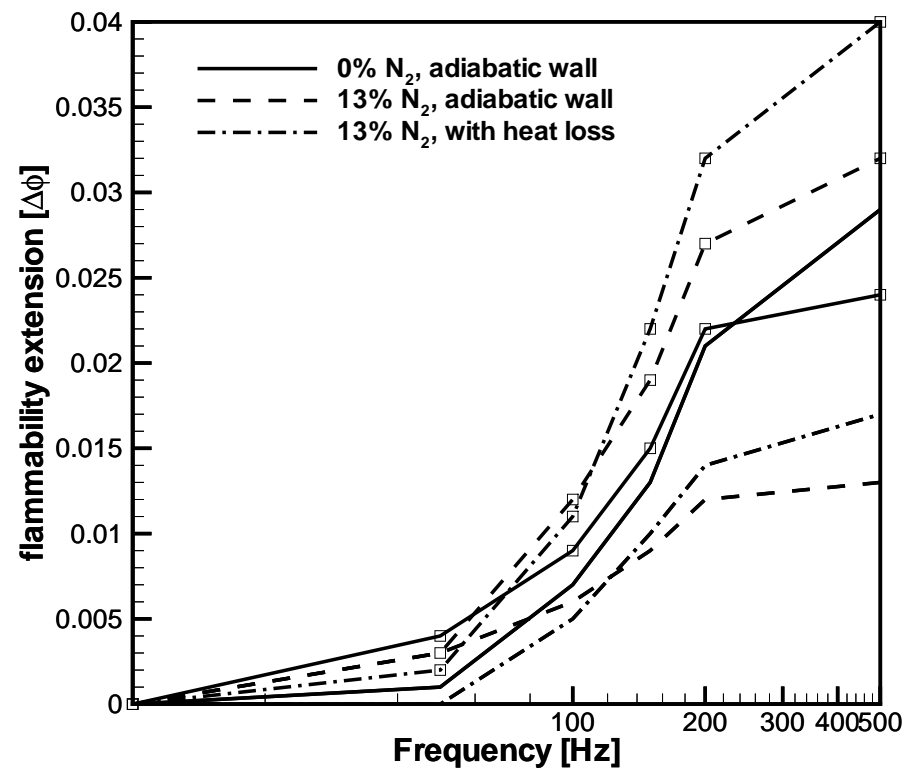

(a)

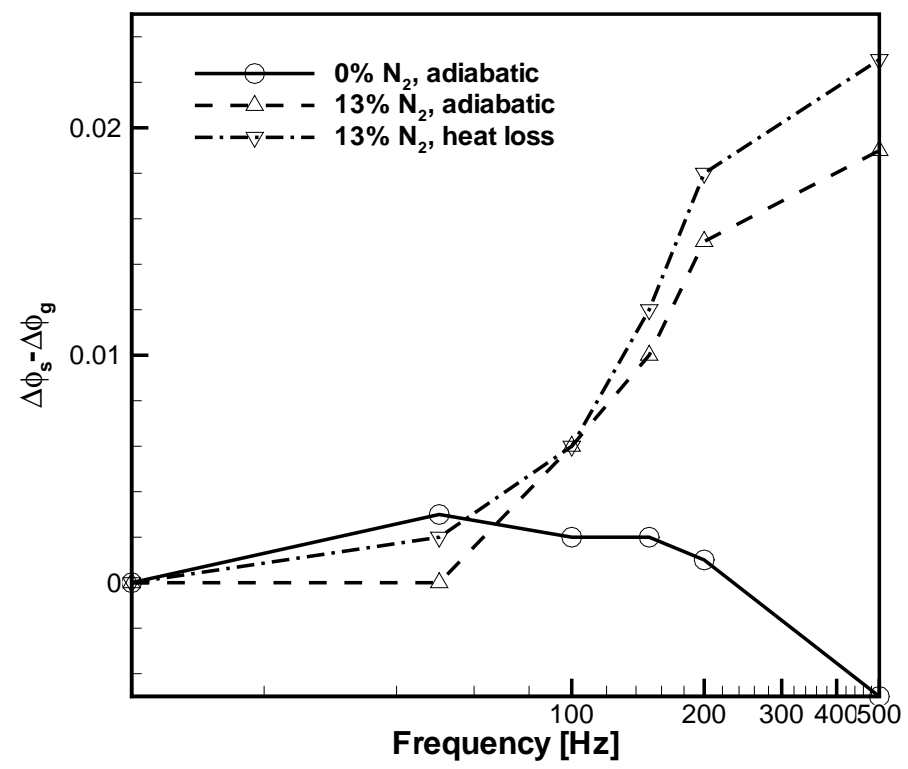

(b)

Figure 5. (a) is Dynamic flammability extension. Lines without square symbols: without surface reactions; lines with square symbols: with surface reactions. (b) is difference between lines with symbol and without symbol in (a). Both (a) and (b) are plotted as a function of frequency, for various dilution and surface thermal conditions. 